\title{
Direct Adaptive Fuzzy Control with Less Restrictions on the Control Gain
}

\section{Phi Anh Phan and Timothy J. Gale}

\begin{abstract}
In the adaptive fuzzy control field for affine nonlinear systems, there are two basic configurations: direct and indirect. It is well known that the direct configuration needs more restrictions on the control gain than the indirect configuration. In general, these restrictions are difficult to check in practice where mathematical models of plant are not available. In this paper, using a simple extension of the universal approximation theorem, we show that the only required constraint on the control gain is that its sign is known. The Lyapunov synthesis approach is used to guarantee the stability and convergence of the closed loop system. Finally, examples of an inverted pendulum and a magnet levitation system demonstrate the theoretical results.
\end{abstract}

Keywords: Affine nonlinear system, direct adaptive fuzzy control, less restriction on the control gain, neural fuzzy system.

\section{INTRODUCTION}

For affine nonlinear systems, adaptive fuzzy control (AFC) can be divided to two categories, direct and indirect. In direct-type schemes, only one neuro-fuzzy system is used as a controller to approximate an ideal control law [1-16]. In the indirect-type, two neurofuzzy systems are used to approximate the non-linear dynamics of the plant [17-22]. Thus, it can be said that direct AFC is structurally simpler than indirect AFC. However, direct $\mathrm{AFC}$ schemes usually require more constraints on the control gain $g(\underline{x})$ (see Section 2 for the definition of $g(\underline{x})$ ) than indirect schemes.

In addition to the well-known necessary condition $g(\underline{x})>0$ for a controllable plant, some extra constraints on $g(\underline{x})$ are needed for stability and convergence analysis. Da and Song [4,5] require that the control gain $g(\underline{x})$ is known. In [2], $g(\underline{x})$ is assumed to be in the form $g(\underline{x})=\frac{1}{c} \bar{g}(\underline{x})$ in which $c>0$ is an unknown scalar constant and $\bar{g}(\underline{x})$ is known. The authors of $[1,6-8]$ require that $g(\underline{x})$ is an unknown constant. In $[10,11]$, the bounds of $g(\underline{x})$ and its first derivative need to be known. In [3], it is assumed that $\frac{\partial g(\underline{x})}{\partial x_{n}}=0$, i.e., the control gain does

Manuscript received December 13, 2006; revised May 25, 2007; accepted August 21, 2007. Recommended by Editorial Board member Young-Hoon Joo under the direction of Editor Jin Young Choi.

Phi Anh Phan, and Timothy J. Gale are with the School of Engineering, University of Tasmania, 64 Water Works Road, Dynnyrne, Hobart, Tasmania, Australia (e-mails: \{paphan, T.Gale\}@utas.edu.au). not depend on the state variable $x_{n}$.

Recently, some researchers have proposed a number of different approaches to relax the extra constraints on $g(\underline{x})$. Wang et al. $[12,13]$ propose a solution in which the control law does not require extra constraint on $g(\underline{x})$. However, $g(\underline{x})$ still needs to be known to implement the adaptive law. Ge et al [3] propose an approach in which they relax extra constraints on $g(\underline{x})$ by using a novel integral-type Lyapunov function. The authors later comment that due to the integral operation, this approach is complicated and difficult to use in practice [14]. Leu et al [15] propose a solution in which the nonlinearity of $g(\underline{x})$ is treated as a component of the overall uncertainty and is cancelled using a variable structure control term. Thus, the bound of $g(\underline{x})$ is still needed. Park et al. [16] propose an approach in which the implicit function theorem is used to solve the problem. A critical step in their design is to determine a constant $c$ such that $c>\frac{1}{2} g(\underline{x})$, thus knowledge of the upper bound of $g(\underline{x})$ is still necessary.

These constraints present difficulties in practice. For instance, the requirement of constant $g(\underline{x})$ restricts the number of plants that direct AFC can be applied to. The requirement of known $g(\underline{x})$ normally requires tests carried on plants to estimate it. Moreover, it cancels out the main advantage of AFthat is no mathematical model of plants is required. Even the requirement of known bound of $g(\underline{x})$ is a disadvantage. If a too conservative bound value is chosen, it usually results in undesired control action. Thus, experiments are also needed to determine the 
bound. These extra experiments add complexity, time and cost to the design of direct AFC.

Why does direct $\mathrm{AFC}$ require more restrictions than indirect AFC in the stability analysis? Are those extra restrictions really necessary conditions? Or are they used simply to overcome obstacles in the stability analysis? We identify that the obstacle lies in the statement of the approximation property of fuzzy logic systems. In this paper, using a simple extension of the universal approximation property, we show that those extra constraints are actually not needed. Based on this property, the stability analysis of direct AFC can be performed very much like its indirect counterpart [1].

\section{PROBLEM STATEMENT}

In this paper, we consider a class of SISO $\mathrm{n}^{\text {th }}$-order affine non-linear systems, which can be represented in the the controllable canonical form.

$$
\begin{aligned}
& \dot{x}_{1}=x_{2}, \\
& \dot{x}_{2}=x_{3}, \\
& \ldots \\
& \ldots \\
& \dot{x}_{n}=f(\underline{x})+g(\underline{x}) u, \\
& y=x_{1},
\end{aligned}
$$

where $u$ is the control input; $y$ is the output; $f(\underline{x})$ and $g(\underline{x})$ are unknown continuous functions; $\underline{x}=$ $\left(x_{1}, x_{2}, \ldots, x_{n}\right)^{T}$ is the state vector of the system, which is assumed available for measurement, and $U_{C}$ is the controllability region.

Control objective: To design an adaptive fuzzy controller such that:

- The closed-loop system is stable in the sense that all the variables in the closed-loop system are bounded.

- The output $y(t)$ of the system follows a continuous reference signal $r(t)$.

To design a controller satisfying the above control objectives, the following assumptions are made:

Assumption 1: $g(\underline{x})$ is continuous and the sign of $g(\underline{x})$ is known for $\underline{x} \in U_{x}$, where $U_{x}$ is the controllability region.

Since $g(\underline{x}) \neq 0$ (controllable condition of system (1)) and $g(\underline{x})$ is continuous for $\underline{x}$ in the controllability region $U_{\underline{x}}$, without loss of generality, it can be assumed that $g(\underline{x})>0$ for $\underline{x} \in U_{\underline{x}}$.

Assumption 2: Define $\underline{r}=\left[\dot{r}, \ddot{r}, \dddot{r} \ldots, r^{(n-1)}\right]^{T}$. We assume that $\|\underline{r}\| \leq r_{0}$ and $\left\|r^{(n)}\right\| \leq r_{1}$ with known constants $r_{0}, r_{1}>0$.

\section{DESCRIPTION OF FUZZY LOGIC SYSTEMS AND THEIR APPROXIMATION PROPERTIES}

In this section, we describe the type of fuzzy logic systems (FLS) used in this paper. Then, we present the universal approximation property of this class of FLSs. The universal approximation property of wider classes of FLSs have already been investigated in the literature [1,2,23-25]. Thus, the purpose of reintroducing the universal approximation property here is to utilize it as a tool to prove Lemma 1 in Section 4 that is necessary for the stability analysis of our proposed controller.

\subsection{Description of fuzzy logic systems}

For each $a<b, a, b \in R$, let $\alpha(a, b): R \rightarrow R$ be a membership function such that $\alpha(a, b)(x) \neq 0$ if $x \in(a, b)$. The chosen fuzzy system has the If-Then rule base of the following form:

$$
\begin{aligned}
\boldsymbol{R}^{(j)}: & \text { IF } x_{1} \text { is } A_{1}^{i} \text {, and } x_{2} \text { is } A_{2}^{i} \text {, and ...and } x_{n} \\
& \text { is } A_{n}^{i} \text {, } \\
& \text { THEN } \text { is } \theta_{j},
\end{aligned}
$$

where $\underline{x}=\left(x_{1}, x_{2}, \ldots, x_{n}\right)^{T} \in U \subset R^{n}$ and $y \in V \subset R$ are the crisp input and output of the fuzzy system. $A_{i}^{j}$ are fuzzy sets with membership functions $A_{i}^{j}\left(x_{i}\right)=\alpha\left(a_{i 1}^{j}, a_{i 2}^{j}\right)\left(x_{i}\right)$ for some $a_{i 1}^{j}<a_{i 2}^{j}, \quad j=1$, $\ldots, M$ where $\mathrm{M}$ is the number of rules, $i=1, \ldots, n$. $\theta_{j}$ is the system output due to rule $R^{(j)}$.

Then, the output of a Takagi-Sugeno fuzzy system is a weighted average of $\theta_{i}$ :

$$
\hat{y}=\hat{f}(\underline{x} \mid \underline{\theta})=\frac{\sum_{j=1}^{M} \theta_{j} \mu_{j}(\underline{x})}{\sum_{j=1}^{M} \mu_{j}(\underline{x})}=\sum_{j=1}^{M} \theta_{j} \zeta_{j}(\underline{x})
$$

in which

$$
\begin{aligned}
& \underline{x}=\left(x_{1}, x_{2}, \ldots, x_{n}\right)^{T} \in U \subset R^{n}, \underline{\theta}=\left(\theta_{1}, \theta_{2}, \ldots \theta_{M}\right)^{T}, \\
& \mu_{j}(\underline{x})=\prod_{i=1}^{n} A_{i}^{j}\left(x_{i}\right), \zeta_{j}(\underline{x})=\frac{\mu_{j}(\underline{x})}{\sum_{j=1}^{M} \mu_{j}(\underline{x})} \geq 0
\end{aligned}
$$

and $\sum_{j=1}^{M} \zeta_{j}(\underline{x})=1, \quad j=1 \ldots M$

3.2. The universal approximation property

The universal approximation property [1,2,23-25] of fuzzy system (2) is stated in Theorem 1 as follows: 
Theorem 1: Let $f: U \subseteq R^{n} \rightarrow R$ be a continuous function defined on a compact $U$, then for each $\varepsilon>0$ there exists a fuzzy system $\hat{f}(\underline{x} \mid \underline{\theta})$ in the form (2) such that

$$
|f(\underline{x})-\hat{f}(\underline{x} \mid \underline{\theta})| \leq \varepsilon, \forall \underline{x} \in U .
$$

Proof: The proof is given in [23].

\section{DIRECT ADAPTIVE FUZZY CONTROL}

To present the control method, first we consider the case where $f(\underline{x})$ and $g(\underline{x})$ in system (1) are known. If we use the controller

$$
u^{*}=\frac{1}{g(\underline{x})}\left(-f(\underline{x})+\underline{k}^{T} \underline{e}+r^{(n)}\right),
$$

where $\underline{e}=\left(e, \dot{e}, \ddot{e}, \ldots, e^{(n-1)}\right)^{T}, e=r-y, \quad \underline{k}=\left(k_{1}, k_{2}\right.$, $\left.\ldots, k_{n}\right)^{T}$, then substituting to (1) gives

$$
e^{(n)}=-\underline{k}^{T} \underline{e}=-k_{1} e-k_{2} \dot{e} \ldots-k_{n} e^{(n-1)} .
$$

We can easily choose $\underline{k}$ such that (4) is Hurwitz stable, i.e., $\lim _{t \rightarrow+\infty} e=0$ or $\lim _{t \rightarrow+\infty} y=r$. Let $v=\underline{k}^{T} \underline{e}$ $+r^{(n)}$. Equation (3) becomes

$$
u^{*}(\underline{X})=\frac{1}{g(\underline{x})}(-f(\underline{x})+v)
$$

in which

$$
\begin{aligned}
& \underline{X}=\left(\underline{x}^{T}, v\right)^{T} \in U_{\underline{X}}, \\
& U_{\underline{X}}=\left\{\underline{X} \mid \underline{x} \in U_{\underline{x}},\|\underline{r}\| \leq r_{0},\left\|r^{(n)}\right\| \leq r_{1}\right\} .
\end{aligned}
$$

Now, consider that $f(\underline{x})$ and $g(\underline{x})$ are not known. We employ a fuzzy logic controller in the form (2)

$$
u=\hat{u}(\underline{X} \mid \underline{\theta})=\sum_{j=1}^{M} \theta_{j} \zeta_{j}(\underline{X})
$$

in which adaptive parameters are the rule consequents $\theta_{j}, j=1 \ldots M$, and $\underline{\theta}=\left(\theta_{1}, \theta_{2}, \ldots \theta_{M}\right)^{T}$. Thus, system (1) becomes

$$
y^{(n)}=f(\underline{x})+g(\underline{x}) \hat{u}(\underline{X} \mid \underline{\theta}) .
$$

Adding and subtracting $g(\underline{x}) u^{*}(\underline{X})$, we have:

$$
\begin{aligned}
y^{(n)}= & f(\underline{x})+g(\underline{x}) \hat{u}(\underline{X} \mid \underline{\theta}) \\
& +g(\underline{x}) u^{*}(\underline{X})-g(\underline{x}) u^{*}(\underline{X}) .
\end{aligned}
$$

From (3),

$$
\begin{aligned}
& y^{(n)}= f(\underline{x})+g(\underline{x})\left(\frac{1}{g(\underline{x})}\left(-f(\underline{x})+\underline{k}^{T} \underline{e}+r^{(n)}\right)\right) \\
&+\left[g(\underline{x}) \hat{u}(\underline{X} \mid \underline{\theta})-g(\underline{x}) u^{*}(\underline{X})\right] \\
& \Leftrightarrow e^{(n)}=-\underline{k}^{T} \underline{e}+\left[g(\underline{x}) u^{*}(\underline{X})-g(\underline{x}) \hat{u}(\underline{X} \mid \underline{\theta})\right] .
\end{aligned}
$$

To continue, we introduce Lemma 1 , which is inspired by the proof of universal property given in [23].

Lemma 1: Given arbitrary $\varepsilon^{*}>0$, there exist $\underline{\zeta}(\underline{X})=\left(\zeta_{1}(\underline{X}), \zeta_{2}(\underline{X}), \ldots \zeta_{M}(\underline{X})\right)^{T}$ and an ideal parameter vector $\underline{\theta}^{*}=\left(\theta_{1}^{*}, \theta_{2}^{*}, \ldots \theta_{M}^{*}\right)^{T}$ such that $g(\underline{x}) u^{*}(\underline{X})-g(\underline{x}) \hat{u}(\underline{X} \mid \underline{\theta})=\sum_{j=1}^{M} c^{j}\left(\theta_{j}^{*}-\theta_{j}\right) \zeta_{j}(\underline{X})+\varepsilon$ where $|\varepsilon| \leq \varepsilon^{*}$ and $c^{j}$ are some positive constants.

Proof: The proof is given in Appendix A.

Applying Lemma 1 to (7), the error dynamic becomes:

$$
e^{(n)}=-\underline{k}^{T} \underline{e}+\left[\sum_{j=1}^{M} c^{j}\left(\theta_{j}^{*}-\theta_{j}\right) \zeta_{j}(\underline{X})+\varepsilon\right] .
$$

In the vector form,

$$
\underline{\dot{e}}=\Lambda_{C} \underline{e}+\underline{b}_{C}\left[\sum_{j=1}^{M} c^{j}\left(\theta_{j}^{*}-\theta_{j}\right) \zeta_{j}(\underline{X})+\varepsilon\right],
$$

where

$$
\Lambda_{\mathrm{C}}=\left(\begin{array}{ccccc}
0 & 1 & 0 & \cdots & 0 \\
0 & 0 & 1 & \cdots & 0 \\
\vdots & \vdots & \vdots & \ddots & \vdots \\
0 & 0 & 0 & \cdots & 1 \\
-\mathrm{k}_{1} & -\mathrm{k}_{2} & -\mathrm{k}_{3} & \cdots & -\mathrm{k}_{\mathrm{n}}
\end{array}\right), \quad \underline{b}_{C}=\left(\begin{array}{c}
0 \\
0 \\
\vdots \\
0 \\
1
\end{array}\right)
$$

Since $\Lambda_{C}$ is a stable matrix, there exists a unique positive definite symmetric $n \times n$ matrix $P$ which satisfies the Lyapunov equation:

$$
\Lambda_{C}^{T} P+P \Lambda_{C}=-Q,
$$

where $Q$ is an arbitrary $n \times n$ positive definite matrix. 
Now, consider the Lyapunov function candidate

$$
V=\frac{1}{2} \underline{e}^{T} P \underline{e}+\frac{1}{2 \gamma} \sum_{j=1}^{M} c^{j}\left(\theta_{j}^{*}-\theta_{j}\right)^{2}
$$

in which $\gamma>0$ is the adaptive gain of the controller. Since $P$ is positive definite and $c^{j}>0, j=1 \ldots M$, it is obvious that $V \geq 0 . \quad \forall \underline{e}, \phi_{u i}$ The time derivative of $V$ along the trajectory of (9) is:

$$
\begin{aligned}
\dot{V}=- & \frac{1}{2} \underline{e}^{T} Q \underline{e}-\frac{1}{\gamma} \sum_{j=1}^{M} c^{j}\left(\theta_{j}^{*}-\theta_{j}\right) \dot{\theta}_{j} \\
+ & \underline{e}^{T} P \underline{b}_{C}\left[\sum_{j=1}^{M} c^{j}\left(\theta_{j}^{*}-\theta_{j}\right) \zeta_{j}(\underline{X})+\varepsilon\right] \\
\Leftrightarrow \dot{V}= & -\frac{1}{2} \underline{e}^{T} Q \underline{e}+\underline{e}^{T} P \underline{b}_{C} \varepsilon \\
& +\frac{1}{\gamma} \sum_{j=1}^{M} c^{j}\left(\theta_{j}^{*}-\theta_{j}\right)\left(\gamma \underline{e}^{T} P \underline{b}_{C} \zeta_{j}(\underline{X})-\dot{\theta}_{j}\right) .
\end{aligned}
$$

If we choose the adaptive law:

$$
\dot{\theta}_{j}=\gamma \underline{e}^{T} P \underline{b}_{C} \zeta_{j}(\underline{X}),
$$

then from (12), we have:

$$
\dot{V}=-\frac{1}{2} \underline{e}^{T} Q \underline{e}+\underline{e}^{T} P \underline{b}_{C} \varepsilon
$$

From Lemma 1, we can expect that $\varepsilon$ should be small, if not equal to zero, provided that we use a sufficient number of rules $M$. If $\varepsilon=0$, then we have $\dot{V}=-\frac{1}{2} \underline{e}^{T} Q \underline{e} \leq 0$. Since $V$ is lower bounded $(\geq 0)$ and $\dot{V}$ is uniformly continuous, using the Barbalat's Lemma, we have $\lim _{t \rightarrow+\infty} \dot{V}=0$, therefore $\lim _{t \rightarrow \infty}|\underline{e}(t)|=0$.

Remark 1: To compensate for the approximation error $\varepsilon$, some authors have proposed different approaches such as using error bound estimation [7,21], $H_{\infty}$ attenuation technique [17], and tuning nonlinear parameters in fuzzy controllers (widths and center of membership functions) [10], etc. We have proposed to use an approximation error estimator in [22]. This approximation error estimator scheme can also be applied to this direct AFC.

Remark 2: To guarantee the boundedness of adaptive parameters, modified adaptive laws such as projection algorithms and $\varepsilon$-modification [1-3,17] can be used. The following algorithm is proposed:

$$
\dot{\theta}_{j}=\left[\begin{array}{cc}
\gamma \underline{e}^{T} P \underline{b}_{C} \zeta_{j}(\underline{X}) \quad \text { if }\left(\theta_{j}^{L}<\theta_{j}<\theta_{j}^{U}\right) \\
& \text { or }\left(\theta_{j}=\theta_{j}^{U} \text { and } \gamma \underline{e}^{T} P \underline{b}_{C} \zeta_{j}(\underline{X})<0\right) \\
& \text { or }\left(\theta_{j}=\theta_{j}^{L} \text { and } \gamma \underline{e}^{T} P \underline{b}_{C} \zeta_{j}(\underline{X})>0\right)(15) \\
0 & \text { if }\left(\theta_{j}=\theta_{j}^{U} \text { and } \gamma \underline{e}^{T} P \underline{b}_{C} \zeta_{j}(\underline{X}) \geq 0\right) \\
& \text { or }\left(\theta_{j}=\theta_{j}^{L} \text { and } \gamma \underline{e}^{T} P \underline{b}_{C} \zeta_{j}(\underline{X}) \leq 0\right)
\end{array}\right.
$$

in which vectors $\underline{\theta}^{L}=\left(\theta_{1}^{L}, \theta_{2}^{L}, \ldots, \theta_{M}^{L}\right)^{T}$ and $\underline{\theta}^{U}=$ $\left(\theta_{1}^{U}, \theta_{2}^{U}, \ldots, \theta_{M}^{U}\right)^{T}$ are chosen such that $\hat{u}\left(\underline{X} \mid \underline{\theta}^{L}\right)$ $\leq u^{*}(\underline{X}) \leq \hat{u}\left(\underline{X} \mid \underline{\theta}^{U}\right), \quad \forall \underline{X} \in U_{\underline{X}}$. Algorithm

guarantees not only that $\theta_{j}^{L} \leq \theta_{j} \leq \theta_{j}^{U}, j=1 \ldots M$, but also $\hat{u}\left(\underline{X} \mid \underline{\theta}^{L}\right) \geq \hat{u}(\underline{X} \mid \underline{\theta}) \geq \hat{u}\left(\underline{X} \mid \underline{\theta}^{U}\right)$. Thus, it allows us to set explicit bounds on the control signal $\hat{u}(\underline{X} \mid \underline{\theta})$. This is useful in practice to avoid actuator saturation and failure.

Remark 3: In the controller, there are 3 design parameters: $\underline{k}, Q$, and $\gamma \cdot \underline{k}$ is chosen such that the error dynamic (4) is Hurwitz stable. Let $\lambda_{\min }(Q)$ be the minimum eigen value of $Q$. In general, increasing $\lambda_{\min }(Q)$ and $\gamma$ lead to better transient response and smaller steady-state error. However, too large $\lambda_{\text {min }}(Q)$ and $\gamma$ lead to chattering and high gain control. Therefore, in practical applications, the design parameters should be adjusted carefully for achieving suitable transient performance and control action.

\section{EXAMPLES}

To demonstrate the theoretical results, we present 2 applications to an inverted pendulum and a magnetic

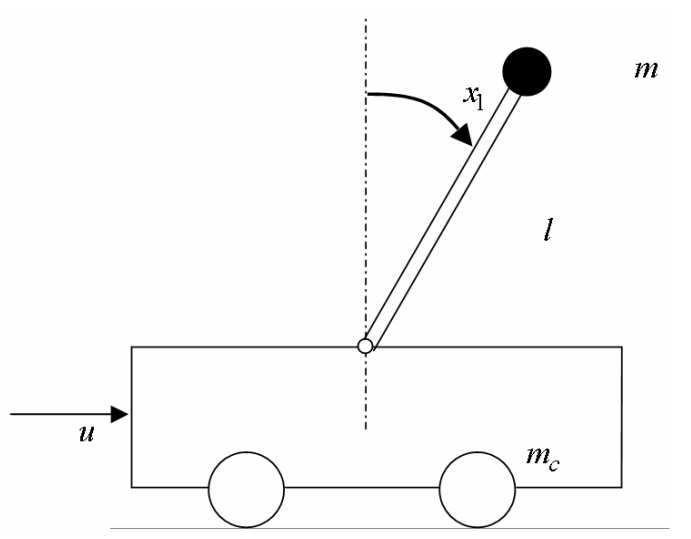

Fig. 1. The inverted pendulum. 
levitation system.

\subsection{Inverted pendulum}

The dynamics of the system (see Fig. 1) is given by:

$$
\begin{aligned}
\dot{x}_{1}= & x_{2}, \\
\dot{x}_{2}= & {\left[g \cdot \sin x_{1}-\frac{m \cdot l \cdot x_{2}^{2} \cdot \cos x_{1} \cdot \sin x_{1}}{m_{c}+m}\right] \cdot \frac{1}{l\left(\frac{4}{3}-\frac{m \cos ^{2} x_{1}}{m_{c}+m}\right)} } \\
& +\frac{\cos x_{1}}{m_{c}+m} \cdot \frac{1}{l\left(\frac{4}{3}-\frac{m \cos ^{2} x_{1}}{m_{c}+m}\right)} \cdot u \\
= & f(\underline{x})+g(\underline{x}) u, \\
y= & x_{1},
\end{aligned}
$$

in which $x_{1}$ is the angular position of the pendulum, $x_{2}$ is the angular velocity of the pendulum, $m_{c}$ is mass of the cart, $m$ is mass of the pendulum and $l$ is half-length of the pendulum. In the simulation, $m_{c}=1 \mathrm{~kg}, m=0.1 \mathrm{~kg}$, and $l=0.5 \mathrm{~m}$. The initial state is $\left[x_{1}(0), x_{2}(0)\right]^{T}=[-\pi / 6,-\pi / 6]^{T}$.

The control objective is to make the angular position $y=x_{1}$ track the reference signal $r(t)=$ $0.5 \sin (t)$.

The operating input ranges are chosen as follows:

$$
x_{1} \in[-1,1] ; x_{2} \in[-1,1] ; v \in[-1,1] \text {. }
$$

The membership functions of each input variable $x_{1}, x_{2}$, and $v$ are chosen as shown in Fig. 2. All possible rules are used. Thus, there are $3 \times 3 \times 3=27$ rules. All the consequent values are initially chosen as zeros.

Assume that, due to the physical limitation of actuators, the force applied on the cart $u$ has to be in the range $[-15 N, 15 N]$. From Remark 2, this can be

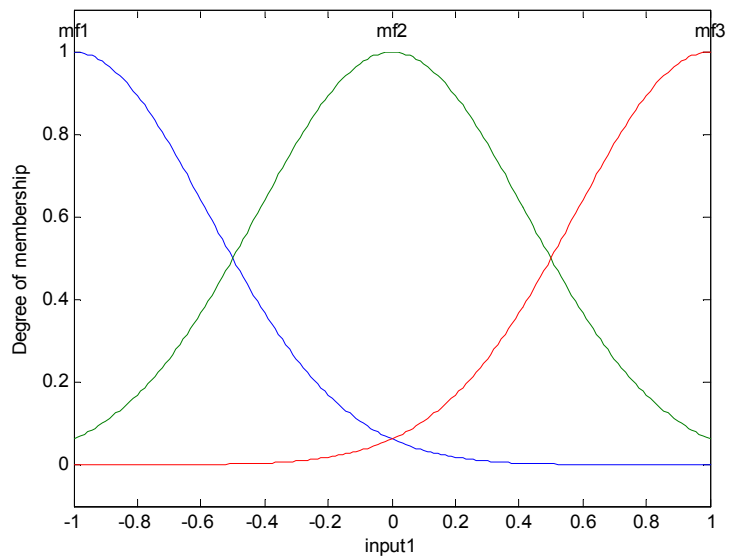

Fig. 2. Membership functions for $x_{1}, x_{2}$, and $v$. assured by setting $\theta_{1}^{L}=\theta_{2}^{L}=\ldots \theta_{27}^{L}=-15$ and $\theta_{1}^{U}=\theta_{2}^{U}=\ldots \theta_{27}^{U}=-15$.

From Remark 3 , the design procedure can be:

- choose $\underline{k}$, and $Q$.

- estimate $P$ using (10).

- tune $\gamma$ until satisfied performance is obtained.

In this application, the controller parameters are chosen as follows:

$$
\underline{k}=\left[\begin{array}{ll}
1 & 1
\end{array}\right]^{T} ; Q=\left[\begin{array}{cc}
20 & 0 \\
0 & 10
\end{array}\right] ; P=\left[\begin{array}{cc}
25 & 10 \\
10 & 15
\end{array}\right] ; \gamma=50 .
$$

The results are shown in Figs. 3-5. It can be seen that the inverted pendulum is successfully controlled by the direct adaptive fuzzy controller. From an initial tracking error of $-\pi / 6$, it converges quickly to the range $[-0.02,0.02]$. The control signal is shown in Fig. 5.

The same application is also controlled successfully in $[5,12]$. However, Gao [5] requires the determination of $g(\underline{x})^{-1}$. In Wang [12], $g(\underline{x})$ needs to be known to implement the adaptive algorithm (28). Also, the bounds of $f(\underline{x})$ and $g(\underline{x})$ are required. Here, we

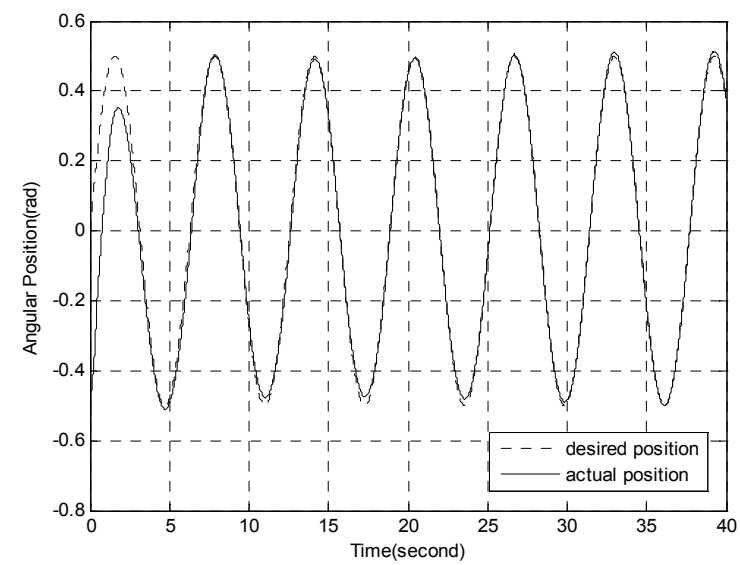

Fig. 3. Angular position.

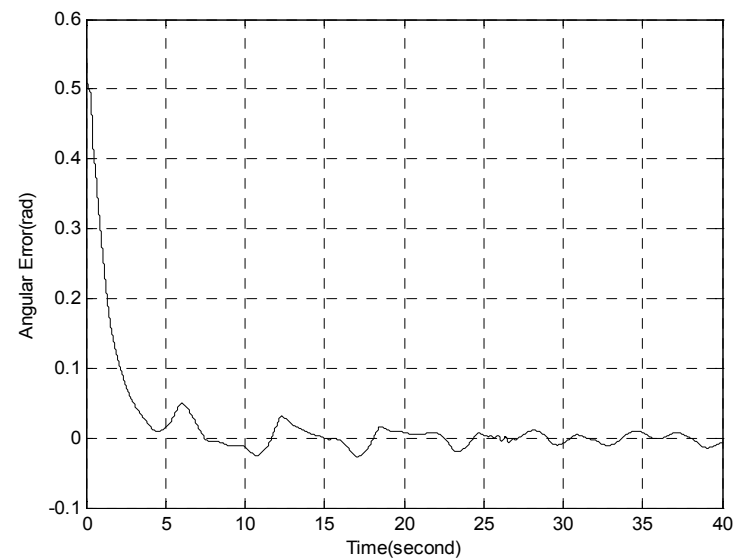

Fig. 4. Tracking error. 


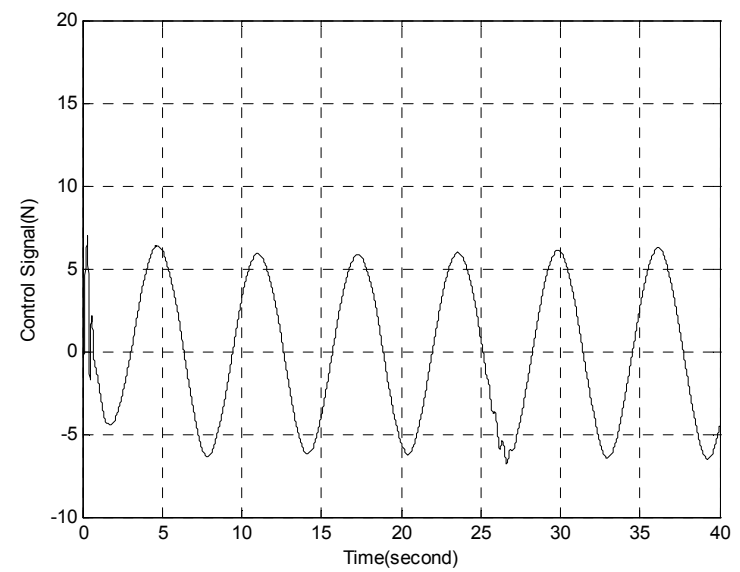

Fig. 5. Control signal.

have shown that the only requirement on the control gain is its sign. This simplifies the design process and eliminates the time and cost of determining those extra requirements.

\subsection{Magnetic levitation system}

In this application, the control objective is to control the position of a magnet suspended above an electromagnet, where the magnet is constrained so that it can only move in the vertical direction (see Fig. 6 ). The equation of motion of this system is:

$$
\ddot{y}(t)=-g+\frac{\alpha}{M} \operatorname{sgn}(i) \frac{i^{2}(t)}{y(t)}-\frac{\beta}{M} \dot{y}(t),
$$

where $y(t)$ is the distance of the magnet above the electromagnet, $i(t)$ is the current flowing in the electromagnet, $M$ is the mass of the magnet, and $g$ is the gravitational constant. The parameter $\beta$ is a viscous friction coefficient that is determined by the material in which the magnet moves, and $\alpha$ is a field strength constant that is determined by the number of turns of wire on the electromagnet and the strength of the magnet. In this application, we choose $M=3 \mathrm{~kg}, \alpha=15$, and $\beta=12$. The desired position

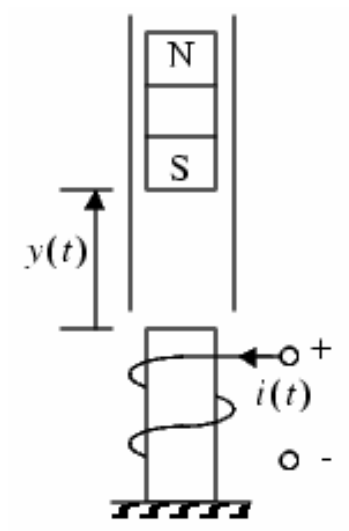

Fig. 6. A magnet levitation system. $y_{d}(t)$ is taken randomly in the range $[0.5 \mathrm{~cm}, 4 \mathrm{~cm}]$. The reference trajectory is generated using a reference model with transfer function $\frac{y_{t}(s)}{y_{d}(s)}=\frac{4}{(s+2)(s+2)}$.

Let $x_{1}=y(t), \quad x_{2}=\dot{y}(t), \quad$ and $\quad u=\operatorname{sgn}(i) i^{2}(t)$. Thus, the current $i$ can be calculated as $i=\operatorname{sgn}(u)$ $\sqrt{a b s(u)}$. The dynamic equations become

$$
\begin{aligned}
& \dot{x}_{1}=x_{2}, \\
& \dot{x}_{2}=-g-\frac{\beta}{M} x_{2}+\frac{\alpha}{M x_{1}} u, \\
& y=x_{1},
\end{aligned}
$$

which is in the affine form (1). Therefore, we can apply our proposed direct AFC to control this system.

The range of the inputs are:

$$
x_{1} \in[0,5] ; \quad x_{2} \in[-5,10] ; \quad v \in[-10,10] .
$$

The membership functions of the three input variables are in Figs. 7-9. All the consequent values are initially chosen as zero.

Assume that the current flowing in the

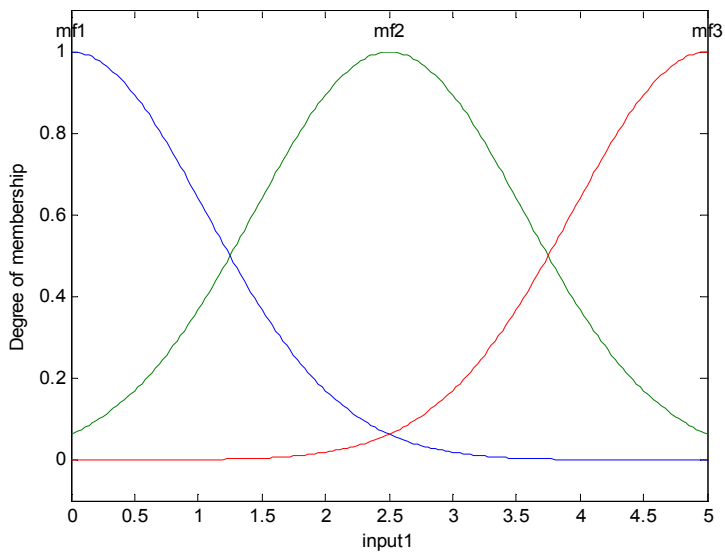

Fig. 7. Membership functions for $x_{1}$.

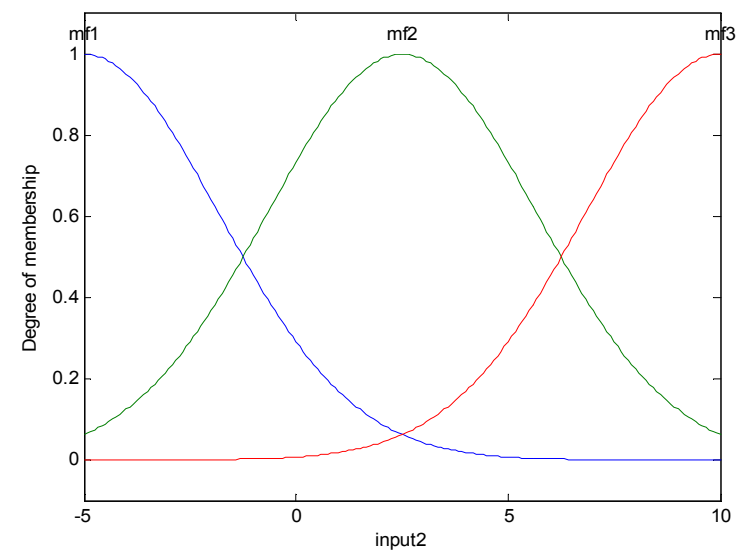

Fig. 8. Membership functions for $x_{2}$. 
electromagnet $i(t)$ has to be in the range $[-5 A, 5 A]$. From Remark 2, this can be easily assured by setting $\theta_{1}^{L}=\theta_{2}^{L}=\ldots \theta_{27}^{L}=-5$ and $\theta_{1}^{U}=\theta_{2}^{U}=\ldots \theta_{27}^{U}=5$.

Using the same design procedure in application 1 , the controller parameters are:

$\underline{k}=\left[\begin{array}{ll}1 & 1\end{array}\right]^{T} ; Q=\left[\begin{array}{cc}20 & 0 \\ 0 & 10\end{array}\right] ; \quad P=\left[\begin{array}{cc}25 & 10 \\ 10 & 15\end{array}\right] ; \gamma=25$.

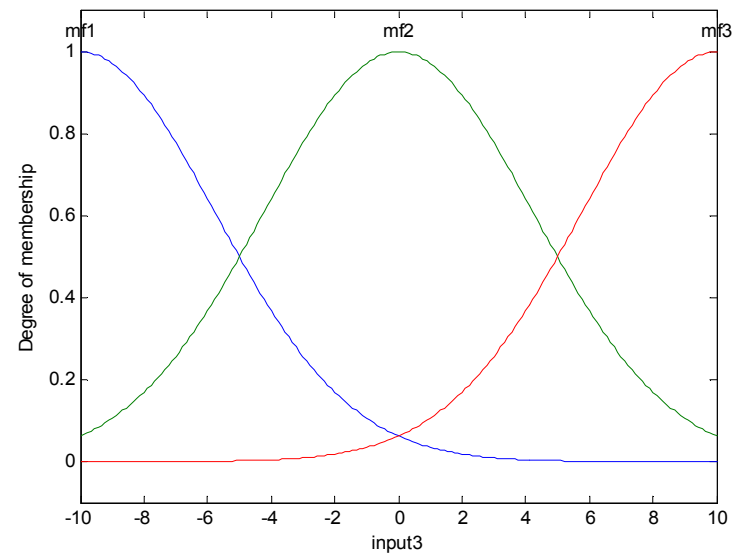

Fig. 9. Membership functions for $v$.

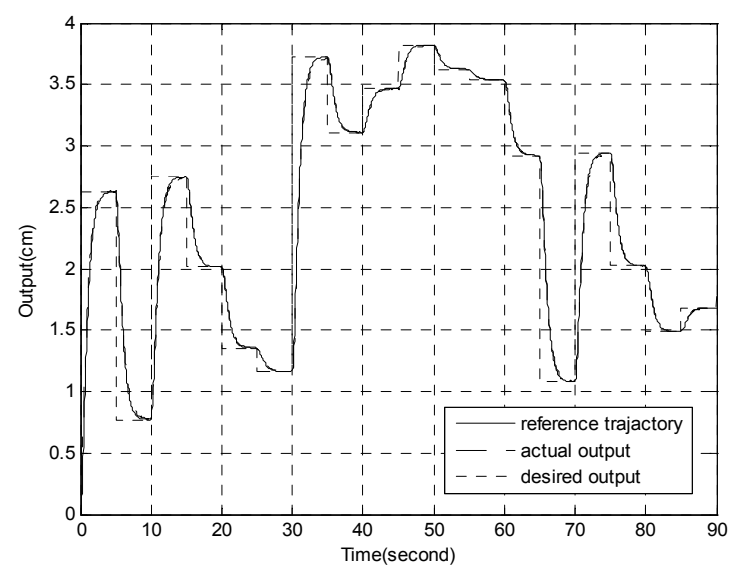

Fig. 10. Output position (cm).

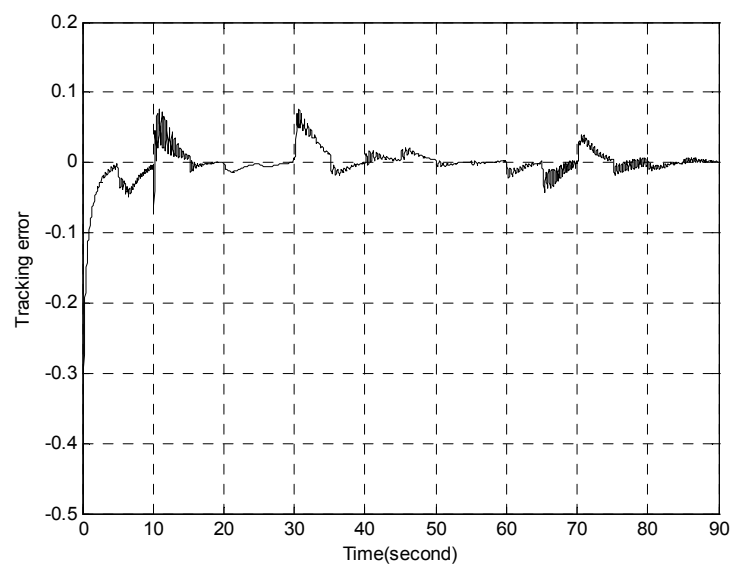

Fig. 11. Tracking error.

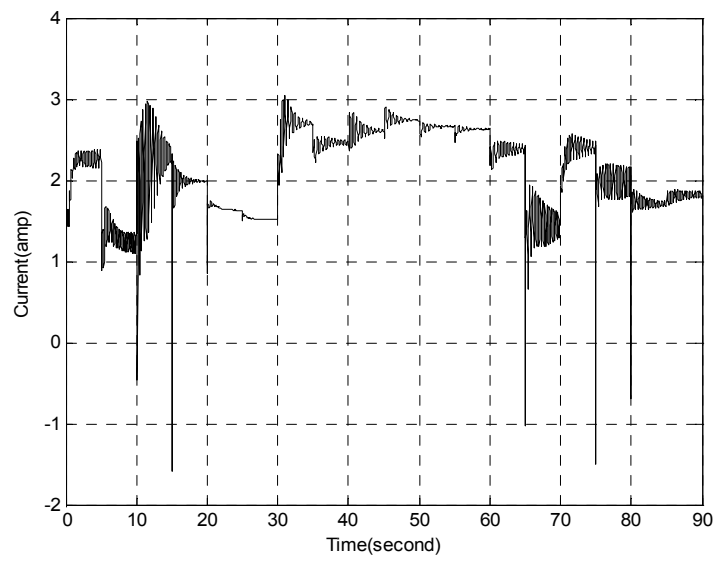

Fig. 12. Control signal.

The results are shown in Figs. 10-12. It can be observed that the actual output tracks closely the reference trajectory. Fig. 11 shows that the tracking error is maintained in the range $[-0.1 \mathrm{~cm}, 0.1 \mathrm{~cm}]$, and the set-point error converges to a very small neighbourhood of zero. Similar to the first application, the only requirement for the control gain $g(\underline{x})$ is its sign, which is positive in this case. Further knowledge of $g(\underline{x})$ or its bounds are not necessary.

\section{CONCLUSION}

In this paper, we show that in direct $\mathrm{AFC}$, the only requirement is that the sign of the control gain is known and extra requirements on the control gain $g(\underline{x})$ are not needed. Using a simple extension of the well-known universal approximation theorem, we perform stability analysis of the direct AFC, and show that the stability analysis is very much like the stability analysis for the traditional indirect AFC. Applications to an inverted pendulum and a magnet levitation system confirm the theoretical results.

\section{APPENDIX A}

Proof of Lemma 1: Let $\underline{X}^{j} \in U_{\underline{X}}$. As $g(\underline{x})$, $\hat{u}(\underline{X} \mid \underline{\theta})$, and $u^{*}(\underline{X})$ are continuous at $\underline{X}^{j}$, for each $i=1 \ldots n, n+1$, there exists a $\delta_{i}^{j}>0$ such that

$$
\begin{aligned}
& \left|X_{i}-X_{i}^{j}\right|<\delta_{i}^{j}(i=1 \ldots n+1) \\
& \Leftrightarrow \mid\left[g(\underline{X}) u^{*}(\underline{X})-g(\underline{X}) \hat{u}(\underline{X} \mid \underline{\theta})\right] \\
& -\left[g\left(\underline{X}^{j}\right) u^{*}\left(\underline{X}^{j}\right)-g\left(\underline{X}^{j}\right) \hat{u}\left(\underline{X}^{j} \mid \underline{\theta}\right)\right] \mid \leq \varepsilon^{*} \\
& \Leftrightarrow \mid\left[g(\underline{X}) u^{*}(\underline{X})-g(\underline{X}) \hat{u}(\underline{X} \mid \underline{\theta})\right] \\
& -g\left(\underline{X}^{j}\right)\left(u^{*}\left(\underline{X}^{j}\right)-\hat{u}\left(\underline{X}^{j} \mid \underline{\theta}\right)\right) \mid \leq \varepsilon^{*}
\end{aligned}
$$




$$
\begin{aligned}
\Leftrightarrow & \mid\left[g(\underline{X}) u^{*}(\underline{X})-g(\underline{X}) \hat{u}(\underline{X} \mid \underline{\theta})\right] \\
& -c^{j}\left(u^{*}\left(\underline{X}^{j}\right)-\hat{u}\left(\underline{X}^{j} \mid \underline{\theta}\right)\right) \mid \leq \varepsilon^{*},
\end{aligned}
$$

where $c^{j}=g\left(\underline{X}^{j}\right)>0$.

Define

$$
\mathrm{O}_{j}=\left\{\underline{X}|| X_{i}-X_{i}^{j} \mid \leq \delta_{i}^{j}(i=1 \ldots n+1)\right\} .
$$

As $U_{\underline{X}}$ is compact, there exists a finite subfamily $\mathrm{O}_{1}, \mathrm{O}_{2}, \ldots, \mathrm{O}_{M}$ such that

$$
U_{\underline{X}} \subseteq \mathrm{O}_{1} \cup \mathrm{O}_{2} \cup \ldots \cup \mathrm{O}_{M} .
$$

Choose

- $A_{i}^{j}\left(X_{i}\right)=\alpha\left(X_{i}^{j}-\delta_{i}^{j}, X_{i}^{j}+\delta_{i}^{j}\right)\left(X_{i}\right), i=1 \ldots n+1$, $j=1 \ldots M$ such that

$$
\begin{aligned}
& \left\{\begin{array}{l}
A_{i}^{j}\left(X_{i}^{k}\right)=1 \text { if } k=j \\
A_{i}^{j}\left(X_{i}^{k}\right)=0 \text { if } k \neq j
\end{array}, j, k=1 \ldots M\right.
\end{aligned}
$$

From (2) and (A.2),

$$
\begin{aligned}
\hat{u}\left(\underline{X}^{j}\right) & =\sum_{k=1}^{M} \theta_{k} \zeta_{k}\left(\underline{X}^{j}\right) \\
& =\theta_{1} \times 0+\theta_{2} \times 0+\ldots \theta_{j} \times 1+\ldots+\theta_{M} \times 0 \\
& =\theta_{j} .
\end{aligned}
$$

Substituting (A.3) and (A.4) to (A.1), we have:

$$
\left|\left[g(\underline{X}) u^{*}(\underline{X})-g(\underline{X}) \hat{u}(\underline{X} \mid \underline{\theta})\right]-c^{j}\left(\theta_{j}^{*}-\theta_{j}\right)\right| \leq \varepsilon^{*} .
$$

As $\quad \zeta_{j}(\underline{X}) \neq 0$ for $\underline{X} \in \mathrm{O}_{j} \quad$ and $\quad \zeta_{j}(\underline{X})=0$ for $\underline{X}$ $\notin \mathrm{O}_{j}$,

$$
\begin{aligned}
(\mathrm{A} .5) \Rightarrow \mid & \mid\left[g(\underline{X}) u^{*}(\underline{X})-g(\underline{X}) \hat{u}(\underline{X} \mid \underline{\theta})\right] \\
& -c^{j}\left(\theta_{j}^{*}-\theta_{j}\right) \mid \zeta_{j}(\underline{X}) \leq \varepsilon^{*} \zeta_{j}(\underline{X}) .
\end{aligned}
$$

Take the summation for $j=1 \ldots M$,

$$
\begin{aligned}
\sum_{j=1}^{M} \mid\left[g(\underline{X}) u^{*}(\underline{X})-g(\underline{X}) \hat{u}(\underline{X} \mid \underline{\theta})\right] \\
\quad-c^{j}\left(\theta_{j}^{*}-\theta_{j}\right) \mid \zeta_{j}(\underline{X}) \leq \sum_{j=1}^{M} \varepsilon^{*} \zeta_{j}(\underline{X})
\end{aligned}
$$

$$
\begin{gathered}
\Leftrightarrow \mid\left[g(\underline{X}) u^{*}(\underline{X})-g(\underline{X}) \hat{u}(\underline{X} \mid \underline{\theta})\right] \sum_{j=1}^{M} \zeta_{j}(\underline{X}) \\
-\sum_{j=1}^{M} c^{j}\left(\theta_{j}^{*}-\theta_{j}\right) \zeta_{j}(\underline{X}) \mid \leq \varepsilon^{*} \sum_{j=1}^{M} \zeta_{j}(\underline{X}) .
\end{gathered}
$$

Since $\sum_{j=1}^{M} \zeta_{j}(\underline{X})=1$, we have:

$$
\begin{aligned}
& \mid\left[g(\underline{X}) u^{*}(\underline{X})-g(\underline{X}) \hat{u}(\underline{X} \mid \underline{\theta})\right] \\
& -\sum_{j=1}^{M} c^{j}\left(\theta_{j}^{*}-\theta_{j}\right) \zeta_{j}(\underline{X}) \mid \leq \varepsilon^{*} .
\end{aligned}
$$

Thus, $g(\underline{x}) u^{*}(\underline{X})-g(\underline{x}) \hat{u}(\underline{X} \mid \underline{\theta})$ can be approximated by $\sum_{j=1}^{M} c^{j}\left(\theta_{j}^{*}-\theta_{j}\right) \zeta_{j}(\underline{X})$ :

$$
\begin{aligned}
& g(\underline{x}) u^{*}(\underline{X})-g(\underline{x}) \hat{u}(\underline{X} \mid \underline{\theta}) \\
& =\sum_{j=1}^{M} c^{j}\left(\theta_{j}^{*}-\theta_{j}\right) \zeta_{j}(\underline{X})+\varepsilon,
\end{aligned}
$$

where $|\varepsilon| \leq \varepsilon^{*}$ and $c^{j}$ are some positive constants.

\section{REFERENCES}

[1] L. X. Wang, Adaptive Fuzzy Systems and Control: Design and Stability Analysis, PrenticeHall, Englewood Cliffs, New Jersey, 1994.

[2] J. T. Spooner, M Maggiore, R Ordonez, and K.M. Passino, Stable Adaptive Control and Estimation for Nonlinear Systems: Neural and Fuzzy Approximator Technique, WileyInterScience, New York, 2002.

[3] S. S. Ge, C. C. Hang, T. H. Lee, and T. Zhang, Stable Adaptive Neural Network Control, pp. 4748, Kluwer Academic Publishers, IBT Global, London, 2002.

[4] F. P. Da and W. S. Song, "Fuzzy neural networks for direct adaptive control," IEEE Trans. on Industrial Electronics, vol. 50, no. 7, pp. 507-513, 2003.

[5] Y. Gao and M. J. Er, "Online adaptive fuzzy neural identification and control of a class of MIMO nonlinear systems," IEEE Trans. on Fuzzy Systems, vol. 11, no. 4, pp. 462-477, 2003.

[6] K. Fischle and D. Schroder, "An improved stable adaptive fuzzy control method," IEEE Trans. on Fuzzy Systems, vol. 7, no. 1, pp. 27-40, 1999.

[7] M. J. Er and S. H. Chin, "Hybrid adaptive fuzzy controllers of robot manipulators with bounds 
estimation," IEEE Trans. on Industrial Electronics, vol. 47, pp. 1151-1160, 2000.

[8] D. L. Tsay, H. Y. Chung, and C. J. Lee, "The adaptive control of nonlinear systems using the Sugeno-type of fuzzy logic," IEEE Trans. on Fuzzy System, vol. 7, no. 2, pp. 225-229, 1999.

[9] T. C. Chai and S. C. Tong, "Fuzzy direct adaptive control for a class of nonlinear systems," Fuzzy Sets and Systems, vol. 103, no. 3, pp. 379-387, 1999.

[10] H. Han, C. Y. Su, and Y. Stepanenko, “Adaptive control of a class of nonlinear systems with nonlinearly parameterized fuzzy approximators," IEEE Trans. on Fuzzy Sets and Systems, vol. 9, no. 2, pp. 315-323, 2001.

[11] N. Essounbouli and A Hamzaoui, "Direct and indirect robust adaptive fuzzy controllers for a class of nonlinear systems," International Journal of Control Automation and Systems, vol. 4, no. 2, pp. 146-154, 2006.

[12] C. H. Wang, H. L. Liu, and T. C. Lin, "Direct adaptive fuzzy-neural control with state observer and supervisory controller for unknown nonlinear dynamical systems," IEEE Trans. on Fuzzy Systems, vol. 10, no. 1, pp. 39-49, 2002.

[13] C. H. Wang, T. C. Lin, T. T. Lee, and H. L. Liu, "Adaptive hybrid intelligent control for uncertain nonlinear dynamical systems," IEEE Trans. on Systems, Man, and Cybernetics: Part B-Cybernetics, vol. 32, no. 5, pp. 583-597, 2002.

[14] S. S. Ge and C. Wang, "Direct adaptive NN control of a class of nonlinear systems," IEEE Trans. on Neural Networks, vol. 13, no. 1, pp. 214-221, 2002.

[15] Y. G. Leu, W. Y. Wang, and T. T. Lee, "Observer-based direct adaptive fuzzy-neural control for nonaffine nonlinear systems," IEEE Trans. on Neural Networks, vol. 16, no. 4, pp. 853-861, 2005.

[16] J. H. Park, S.-H. Huh, S.-H. Kim, S.-J. Seo, and G.-T. Park, "Direct adaptive controller for nonaffine nonlinear systems using selfstructuring neural networks," IEEE Trans. on Neural Networks, vol. 16, no. 2, pp. 414-422, 2005.

[17] Y. C. Chang, "Adaptive fuzzy-based tracking control for nonlinear SISO systems via VSS and $\mathrm{H}^{\infty}$ approaches," IEEE Trans. on Fuzzy Systems, vol. 9, no. 2, pp. 278-292, 2001.

[18] Y. Diao and K. M. Passino, "Stable faulttolerant adaptive fuzzy/neural control for a turbine engine," IEEE Trans. on Control Technology, vol. 9, no. 3, pp. 494-509, 2001.

[19] Y. X. Diao and K. M. Passino, "Adaptive neural/fuzzy control for interpolated nonlinear systems," IEEE Trans. on Fuzzy Systems, vol. 10, no. 5, pp. 583-595, 2002.

[20] R. Ordonez and K. M. Passino, "Stable multiinput multi-output adaptive fuzzy neural control," IEEE Trans. on Fuzzy Systems, vol. 7, no. 3, pp. 345-353, 1999.

[21] F. C. Sun, Z. Q. Sun, Y. Y. Zhu, and W. J. Lu, "Stable neuro-adaptive control for robots with the upper bound estimation on the neural approximation errors," Journal of Intelligent \& Robotic Systems, vol. 26, no. 1, pp. 91-100, 1999.

[22] P. A. Phan and T. Gale, "Two-mode adaptive fuzzy control with approximation error estimator," IEEE Trans. on Fuzzy Systems, vol. 15, no. 2, pp. 943-955, 2007.

[23] J. L Castro, "Fuzzy logic controllers are universal approximators," IEEE Trans. on Systems, Man and Cybernetics, vol. 25, no. 4, pp. 629-635, 1995.

[24] B. Kosko, "Fuzzy systems as universal approximatiors," IEEE Trans. on Computers, vol. 4, pp. 1329-1333, 1994.

[25] X. J. Zeng and M. G. Singh, "Approximation theory of fuzzy systems-SISO case," IEEE Trans. on Fuzzy Systems, vol. 2, no. 2, pp. 162176, 1994.

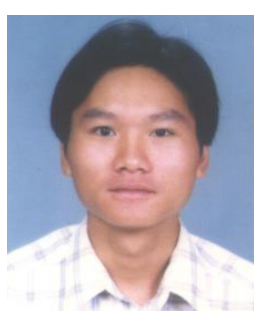

Phi Anh Phan received the B.Eng. degree in Mechatronics from the University of Tasmania, Australia in 2002. He is currently working toward a Ph.D. degree in the School of Engineering at the same university. His research interests are intelligent systems, intelligent control, nonlinear control, and robotics.

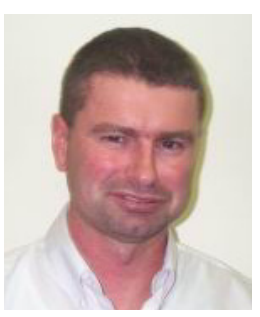

Timothy J Gale obtained the Masters degree from the University of Melbourne in 1991, and the Ph.D. degree in Biomedical Engineering from the University of Tasmania in 1996. He currently coordinates and teaches in the Mechatronic Engineering Degree program at the University of Tasmania. His research interests include modeling, AI, control and medical device development. 